\title{
Feasibility of home and hospital colorectal irrigation with continuous tube placement for Hirschsprung's disease in neonates and infants: a comparative retrospective study
}

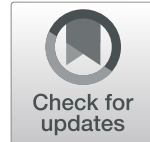

Tsuyoshi Sakurai* (D), Megumi Nakamura and Naobumi Endo

\begin{abstract}
Background: Since the length of the affected colon widely varies, the preoperative management of Hirschsprung's disease varies from one hospital to another. For our cases in which the length of the aganglionic colon cannot be managed by enema, anal bougienage, or rectal irrigation, colorectal irrigation is used along with the placement of a transanal catheter for these patients as home or hospital management, until one-stage definitive surgery can be performed. No comparative studies have been conducted on the continuous tube placement method, and no study has utilized this method as home management. Thus, this study aimed to analyze the efficacy and feasibility of our proposed continuous tube placement management for patients with Hirschsprung's disease in the hospital or even at home.
\end{abstract}

Results: We included 22 infants with Hirschsprung's disease between 2008 and 2018. The patients were divided into two groups: those who were managed with enema, bougienage, and rectal irrigation $(n=6)$, and those who were managed with the placement of a continuous transanal tube and colorectal irrigation $(n=16)$. The group with continuous transanal tube placement was further divided into two groups: those who were preoperatively managed with a continuous anorectal tube at home $(n=7)$, and those at a hospital $(n=9)$. Preoperative demographic information and clinical details were retrospectively examined and compared between the two groups. Univariate analysis showed no significant differences in the backgrounds and clinical status between the enema, bougienage, and rectal irrigation group and the colorectal irrigation group. The patients in the home group were older and weighed more at the time of radical surgery than those in the hospital group $(p=0.0267, p=$ 0.0377 , respectively). The total duration of hospitalization until radical surgery was significantly decreased in the home group ( $p=0.0315$ ).

Conclusions: The total duration of hospitalization was significantly reduced in patients undergoing home colorectal irrigation which was as effective as the conventional method, with no impact on the preoperative condition or postoperative outcomes. Hence, our home-based preoperative management for patients with Hirschsprung's disease may be effective and potentially feasible for the management of patients with Hirschsprung's disease.

Keywords: Anorectal tube, Hirschsprung's disease, One-stage surgery, Preoperative, Rectal irrigation

*Correspondence: sakurai@ped-surg.med.tohoku.ac.jp

Department of Pediatric Surgery, Miyagi Children's Hospital, Sendai 989-3126,

Japan

(c) The Author(s). 2021 Open Access This article is licensed under a Creative Commons Attribution 4.0 International License, which permits use, sharing, adaptation, distribution and reproduction in any medium or format, as long as you give appropriate credit to the original author(s) and the source, provide a link to the Creative Commons licence, and indicate if changes were made. The images or other third party material in this article are included in the article's Creative Commons licence, unless indicated otherwise in a credit line to the material. If material is not included in the article's Creative Commons licence and your intended use is not permitted by statutory regulation or exceeds the permitted use, you will need to obtain permission directly from the copyright holder. To view a copy of this licence, visit http://creativecommons.org/licenses/by/4.0/. 


\section{Background}

Hirschsprung's disease (HD) is characterized by the absence of ganglion cells in the myenteric and submucosal plexuses of the intestine. Poor intestinal peristalsis due to the aganglionic intestine leads to abdominal distention, biliary vomiting, and Hirschsprung-associated enterocolitis (HAEC) as a fatal complication. To decompress the oral side of the aganglionic intestine and prevent the development of complications, colostomy or ileostomy were recommended. However, in the 1980s, many surgeons reported a series of one-stage pullthrough procedures [1, 2], and the one-stage definitive surgery for patients with HD without ostomy has gained widespread acceptance among surgeons [3]. To prevent the development of complications after this one-stage definitive operation, appropriate preoperative management with enemas, anal bougienage, and rectal irrigations is required, instead of ostomy.

Hirose et al. first reported a method of transanal catheter fixation and colonic irrigation for the preoperative management of neonates with HD [4]. Mochizuki et al. also reported that this procedure was safe and effective even for long- and total-type HD [5]. This method enables intestinal decompression for a relatively long period; thus, the radical surgery can be made semielectively. However, to the best of our knowledge, no comparative studies have been conducted on this method. Thus, we evaluated the feasibility of this continuous tube placement for HD by comparing with other methods of preoperative management.

Besides, this technique has some issues, such as prolonged hospitalization and mother-infant separation. Lu et al. [6] reported that rectal irrigation at home was feasible and effective in neonates and infants with HD; however, due to factors like an extended aganglionic segment, older age, or high HAEC score at the time of diagnosis of $\mathrm{HD}$, rectal irrigation may be not suitable. These cases could be solved by making our colorectal irrigation method home-managed, because more proximal parts of the colon can be managed effectively with this treatment compared to localized treatment with the rectal irrigation. We hypothesized that this method could be safely carried out even at home if sufficient parents' education and emergency response were provided. In this study, we aimed to demonstrate the efficacy and feasibility of colorectal irrigation technique, whether in the hospital or home colorectal irrigation (HCI) technique.

\section{Methods}

The feasibility of colorectal irrigation with the continuous tube placement method

At our institution, patients with suspected HD are initially managed with a glycerin enema, finger bougienage and, sometimes, rectal irrigation. Decompression with a continuous transanal tube is required when HD cannot be managed with conventional methods until radical surgery is performed. If decompression cannot be done, or if the surgery is delayed due to complications such as cardiac malformations, the patients need ileostomy or colostomy.

In this retrospective study, we enrolled patients with confirmed HD who were being followed up at the outpatient clinic of our institution from 2008 to 2018. We enrolled 28 patients with confirmed HD who underwent radical surgery during infancy (median age at definitive surgery, 1.6 months; female, $n=4$; male, $n=28$ ). We excluded two patients who had undergone radical surgery in other hospitals and four who needed ileostomy or colostomy. Finally, 22 patients were included in our study; they were then divided into the following two groups: those who were managed with conventional methods $(n=6)$, and those who were managed with the placement of a continuous transanal tube and colorectal irrigation $(n=$ 16). The colorectal irrigation group was divided into the following two groups: those who were managed with colorectal irrigation at home with the anorectal tube ( $\mathrm{HCI} ; n=$ 7 ) and those who were managed at the hospital (non-HCI; $n=9$ ) (Fig. 1). The choice of management location mainly depended on the family's preference. The medical records of each patient were retrospectively reviewed for clinical details, such as sex, gestational age, birth weight, cardiac malformations, Trisomy 21, type of HD (either shortsegment type HD that is defined as the presence of aganglionosis up to the sigmoid colon or long-segment type $\mathrm{HD}$ that is defined as the presence of aganglionosis extending proximally beyond the sigmoid colon). Also, age and weight at radical surgery, duration from rectal mucosal biopsy to surgery, operative time, postoperative stenosis of the anal canal, pre-HAEC before the managements and postoperative HAEC, and follow-up duration, to compare the patients' characteristics between the $\mathrm{HCI}$ and non-HCI groups. All patients underwent a transanal endorectal pull-through surgery with rectoanal myotomy and rectoplasty with or without laparotomy or laparoscopic assistance [7]. HAEC was defined as a condition obtaining a score of at least four according to the guidelines described by Frykman et al. [8] and requiring hospitalization. In addition, to evaluate the feasibility of the HCI technique with transanal tube placement, we compared the age at tube insertion, duration of tube placement, times of tube exchanges, total duration of hospitalization until definitive surgery, and total duration of hospitalization until radical surgery from the time of tube insertion between the two groups.

\section{Continuous tube placement treatment in the colorectal irrigation technique and its home management} An 8-12-Fr soft drainage tube (Salem Sump ${ }^{\text {тм }}$ Tube, Japan Coviden) or an 8-Fr balloon catheter (Japan Create 


Patients with confirmed HD who were being followed up
between 2008 and 2018

Fig. 1 Patient selection flowchart

Medic) was inserted into the rectum under fluoroscopic guidance and was advanced to the dilated colon (Fig. 2). The method of fixation to the patient's hip was according to the technique described previously [5]. The tube was tied with a thread and was fixed by applying a tape to the hip using the thread (Fig. 3). For colorectal irrigation, approximately $20 \mathrm{~mL}$ of normal saline was repeatedly injected via the tube until the collected saline contained almost no stool. The patients' parents were instructed on how to perform colorectal irrigation by pediatric surgeons and nurses for approximately 2-3 days. They were also informed that, if the irrigation was not performed appropriately, the infant would have

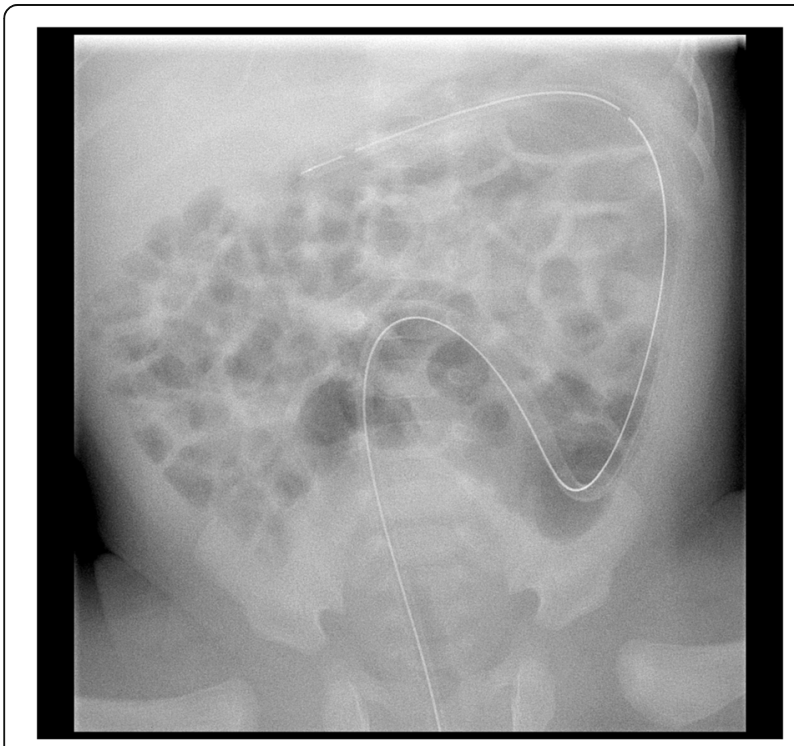

Fig. 2 Contrast enema showing the type of anorectal tube placed in the transverse colon abdominal distension or would appear unwell or lethargic. We ensured that they could easily contact the hospital or have access to our hospital, at any time. After the patient was discharged from the hospital, their parents performed colorectal irrigation twice daily at home. The tube replacement frequency at the hospital was approximately 14 days, if there was no unplanned removal. After a definitive histological confirmation $[9,10]$, radical surgery was performed when the patient did not have a dilated intestinal tract and had a good nutritional status.

\section{Patient consent and ethical committee agreement}

This study was approved by the ethics committee of Miyagi Children's Hospital (protocol identification number: 497). Written informed consent was obtained from the parents.

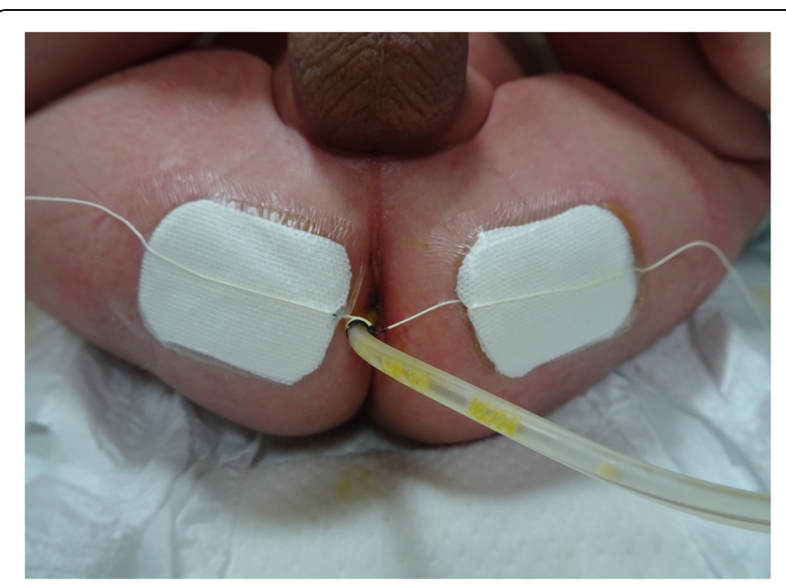

Fig. 3 The tube fixation on the patient's hip 


\section{Statistical analysis}

For the statistical comparison of the data between the two groups, the data obtained in this study were assessed using the $t$ test or Wilcoxon rank sum test (non-parametric test) depending on whether the data were normally distributed or not. All statistical analyses in this study were performed using JMP ${ }^{\circ}$ Pro 14 (SAS Institute Japan Ltd.). Statistical significance was determined as $p$ $<0.05$.

\section{Results}

To evaluate the validity and safety of colorectal irrigation with continuous tube management, we compared the patient's backgrounds, the patient's age, and weight at the time of surgery, operating time, and postoperative complications between the conventional management group and colorectal irrigation management groups. There were no significant differences in the backgrounds; the age of the patients at the time of surgery and operating time; and the postoperative complications between the two groups. Both groups had no symptoms due to dilated intestinal tract such as enteritis or abdominal distension after the start of management (Table 1).

Second, we examined the feasibility of at-home colorectal irrigation management, which is non-inferior to existing preoperative management method. Table 2 summarizes the demographic details of the patients in both $\mathrm{HCI}$ and non-HCI groups. The univariate analysis revealed no significant differences in the preoperative status with respect to sex, gestational age, birth weight, age at diagnosis, type of HD, preoperative enterocolitis, and other complications between the $\mathrm{HCI}$ and non-HCI groups. The patients in the $\mathrm{HCI}$ group were older and weighed more at the time of radical surgery than those in the non- $\mathrm{HCI}$ group (median, 56 versus 34 days, $p=$ 0.0267 ; median, 4.5 versus $3.8 \mathrm{~kg}, p=0.0377$, respectively). Surgical time and frequency of postoperative complications, postoperative anal canal stenosis, and postoperative enterocolitis were not significantly different between the two groups.

Additionally, we compared the factors affected by tube placement between the non-HCI and HCI groups (Table 3) in detail. The total duration of hospitalization until radical surgery and the time from tube insertion until radical surgery were significantly shorter in the non-HCI group than in the $\mathrm{HCI}$ group (median, 48 [non- $\mathrm{HCI}]$ versus $21[\mathrm{HCI}]$ days, $p=0.0315$; median, $48[$ non- $\mathrm{HCI}]$ versus $21[\mathrm{HCI}]$ days, $p=0.0129$, respectively). The age at the time of tube insertion, duration of tube insertion, and times of tube exchanges were not significantly different between the two groups. In the HCI group, the median duration of $\mathrm{HCI}$ management was 21 days.

\section{Discussion}

In this study, we investigated the efficacy and feasibility of the preoperative colorectal irrigation at home and in the hospital with continuous tube placement in patients with HD. No differences were found in the demographic characteristics or postoperative outcomes between the group that was treated by enema, anal bougienage, or rectal irrigation, and the group that was treated by colorectal irrigation. Additionally, there were no differences in the demographic characteristics or postoperative outcomes between at-home and hospital colorectal irrigation management; hence, the $\mathrm{HCI}$ method could be considered as safe as hospital management. We also

Table. 1 Comparison of background and perioperative factors between the conventional management group and tube management groups

\begin{tabular}{llll}
\hline & Conventional $(\boldsymbol{n}=\mathbf{6})$ & Colorectal irrigation $(\boldsymbol{n}=\mathbf{1 6})$ & $\boldsymbol{p}$ value \\
\hline Sex (F:M) & $2: 4$ & $2: 14$ & 0.292 \\
Gestational age (weeks) & $38.9[37,41]$ & $38.5[36.1,41.1]$ & 0.867 \\
Birth weight (kg) & $2.93[2.44,3.59]$ & $2.25[2.25,4.09]$ & 0.843 \\
Cardiac malformations & $1(17 \%)$ & $3(19 \%)$ & 1.0 \\
Trisomy 21 & $1(17 \%)$ & $3(19 \%)$ & 1.0 \\
Type of HD (short: long) & $6: 0$ & $13: 3$ & $6(38 \%)$ \\
Preoperative enterocolitis & $2(33 \%)$ & $15.5[6,31]$ & 0.533 \\
Interval from biopsy to surgery (days) & $14.5[6,36]$ & $44.5[18,104]$ & 1.0 \\
Age at radical surgery (days) & $48[20,86]$ & $4.0[2.9,5.6]$ & 0.617 \\
Weight at radical surgery (kg) & $4.4[2.8,5.2]$ & $293[145,487]$ & 0.913 \\
Surgical time (min) & $221[162,289]$ & $2(13 \%)$ & 0.546 \\
Postoperative stenosis & $1(17 \%)$ & $6(38 \%)$ & 0.0684 \\
Postoperative enterocolitis & $1(17 \%)$ & 1.0 \\
\hline Data & 0.616 \\
\hline
\end{tabular}

Data are presented as frequency (\%) for qualitative parameters and median [range] for continuous parameters. Statistical significance was determined as $p<0.05$ $F$ female, $M$ male, $H D$ Hirschsprung's disease 
Table. 2 Comparison of background and perioperative factors between the non- $\mathrm{HCl}$ and $\mathrm{HCl}$ groups

\begin{tabular}{|c|c|c|c|}
\hline & Non-HCl $(n=9)$ & $\mathrm{HCl}(n=7)$ & $p$ value \\
\hline $\operatorname{Sex}(F: M)$ & $1: 6$ & $1: 8$ & 0.849 \\
\hline Gestational age (weeks) & $39[38,40.9]$ & $38[36.6,41.1]$ & 0.183 \\
\hline Birth weight (kg) & $3.21[2.25,4.06]$ & $2.95[2.44,3.42]$ & 0.669 \\
\hline Cardiac malformations & $1(14 \%)$ & $2(22 \%)$ & 0.0833 \\
\hline Trisomy 21 & $0(0 \%)$ & $3(33 \%)$ & - \\
\hline Type of HD (short: long) & $6: 1$ & $7: 2$ & 0.687 \\
\hline Preoperative enterocolitis & $4(44 \%)$ & $2(29 \%)$ & 0.633 \\
\hline Interval from biopsy to surgery (days) & $15[8,20]$ & $21[6,31]$ & 0.248 \\
\hline Age at radical surgery (days) & $34[18,49]$ & $56[44,105]$ & $0.0267^{*}$ \\
\hline Weight at radical surgery (kg) & $3.8[2.9,4.6]$ & $4.5[3.6,5.6]$ & $0.0377^{*}$ \\
\hline Surgical time (min) & $297[236,487]$ & $248[145,412]$ & 0.234 \\
\hline Postoperative stenosis & $2(22 \%)$ & $0(0 \%)$ & - \\
\hline Postoperative enterocolitis & $2(22 \%)$ & $4(57 \%)$ & 0.152 \\
\hline
\end{tabular}

Data are presented as frequency (\%) for qualitative parameters and median [range] for continuous parameters. Statistical significance was determined as $p<0.05$ ) $H R I$ home rectal irrigation, $F$ female, $M$ male, $H D$ Hirschsprung's disease ${ }^{*} p<0.05$

noted that the total duration of hospitalization could be shortened with $\mathrm{HCI}$; thus, we suggest that the $\mathrm{HCI}$ method was effective.

HD can cause HAEC, which is a life-threatening complication, both before and after surgery [11]; thus, it is important for patients and their families to understand how to prevent HAEC. It is also necessary to restore the dilated intestine to its normal size for radical surgery. Hence, most surgeons usually opt for glycerin enemas, rectal irrigation, or ostomies as the preoperative management of HD. The European Reference Networks guidelines recommend that patients with HD receive saline rectal irrigations 1-3 times per day to decompress the bowel until the definitive pull-through operation [12]. However, in cases that cannot be managed with a glycerin enema, finger bougienage, or rectal irrigation, an ostomy is performed to prevent HAEC. We chose colorectal irrigation with continuous tube placement as the preoperative management in these cases, even in those with long-type HD. The tube insertion technique poses a risk of rectosigmoid colon injury and perforation, especially in neonates and infants [13]. We mainly used a 8-12-Fr soft drainage tube (Salem Sump ${ }^{\text {Tm }}$ Tube, Japan Coviden), as the decompression efficiency of this tube is better, and the shape of the tip is relatively round compared to other available tubes; this minimizes the risk of perforation. Furthermore, to prevent perforation of the colon, under fluoroscopic guidance, the tip is placed beyond the aganglionic segment to the middle of the descending colon or transverse colon so that the tip is not placed at the flexion of the colon, such as the sigmoid colon flexion. In the present study, no HAEC occurred after tube insertion in any patient, including those with long-type HD. Additionally, the colorectal irrigation method was not significantly different from the conventional method in terms of intraoperative and postoperative results, suggesting that it is a safe and effective method for the management of HD.

After tube placement, the patients with HD were preoperatively managed at home or at the hospital,

Table. 3 Comparison of tube insertion characteristics between the non- $\mathrm{HCl}$ and $\mathrm{HCl}$ groups

\begin{tabular}{llll}
\hline & Non- $\mathbf{H C l}(\boldsymbol{n}=\mathbf{9})$ & $\mathbf{H C l}(\boldsymbol{n}=\mathbf{7})$ & $\mathbf{p}$ value \\
\hline Total duration of hospitalization until radical surgery (days) & $48[25,108]$ & $21[11,61]$ & $0.0315^{*}$ \\
Total duration of hospitalization until radical surgery from tube insertion (days) & $48[22,108]$ & $21[11,38]$ & $0.0129^{*}$ \\
Age at tube insertion (days) & $13[1,44]$ & $23[2,68]$ & 0.222 \\
Duration of tube insertion (days) & $22[17,37]$ & $34[15,78]$ & $3[1,5]$ \\
Times of tube exchange procedures & $2[1,3]$ & - & $21[4,48]$ \\
Duration of HRI (days) & - & -320 \\
\hline
\end{tabular}

Data are presented as median [range] for continuous parameters. Statistical significance was determined as $p<0.05$ $H R I$ home rectal irrigation

${ }^{*} p<0.05$ 
according to the family's preference. Although the preference of the family may have been highly biased, there was no difference in patient background between the $\mathrm{HCI}$ and non-HCI groups. The consent was made with respect for the thoughts of the patient's family, so that they could consider the disadvantages of HD management at both locations, which were extension of the hospitalization period and the added costs of hospital management; or anxiety about management at home, before making an informed consent. The parents may have been anxious when doing the procedure, since a lot of effort and time were required, as the irrigation had to be performed twice a day. However, in the present study, none of the patients suffered perforation of the colon, and the surgical results in the $\mathrm{HCI}$ and non-HCI groups were similar.

In our study, patients in the HCI group were significantly older and weighed more at the time of radical surgery than those in the non-HCI group. After tube placement, radical surgery was performed as a semielective surgery because stable intestinal decompression was possible, and the patients did not require early radical surgery. On the other hand, the non-HCI group tended to undergo surgery and be discharged as soon as possible to avoid maternal separation and long-term hospitalization. Hence, it was possible that the radical surgery was delayed in the HCI (outpatient) group compared to that in the non-HCI (inpatient) group. Although the optimal timing of radical surgery for HD patients has not been determined to date, no specific advantages have been identified for performing pullthrough surgery at an early age, such as during the neonatal period [12]. However, Lu et al. [14] showed that radical surgery performed in the neonates rather than the non-neonates was accompanied by complications, such as perianal inflammation, anastomotic strictures or leakage, and HAEC. Hence, even if HCI delays surgery, the delay may be reasonable. Our results did not find a significant difference in the surgical time or postoperative complications, other than the age and weight of the patients at the time of surgery, between the two groups.

The time taken for the tube exchange procedure did not significantly increase in the HCI group, and the total duration of hospitalization was shortened in the $\mathrm{HCI}$ group, thereby reducing the hospitalization costs. Moreover, home management may potentially have an additional advantage of eliminating the need for separating the mothers from their infants who are generally admitted in the neonatal intensive care unit.

Our study had some limitations. The selection of the indications for this method was difficult. Whether or not patients with the HD patients are uncontrollable cases in the conventional methods can be subjective estimation, and our method might be over-adapted. Besides, since three of the four stoma management patients in this study had total colon aganglionosis, this method may not be applicable if the aganglionic colon extends to the entire colon. Additionally, the data were based on subjective responses of the families of the patients, which could compromise the quality of the data owing to some biases. We offered in-patient management if the patient's family was not confident in managing the condition with HCI. Parents who refuse HCI had a strong sense of anxiety about home management, which may have led to earlier surgery being performed at a younger age in the non-HCI group. In addition, this method could increase infants' exposure to radiation, and it could take time and effort for parents to learn to perform home management for their infants with tubes.

\section{Conclusions}

Preoperative management for HD patients by continuous tube placement method can be performed without major problems, and is just as safe as the conventional method. The total duration of hospitalization was significantly reduced in patients undergoing $\mathrm{HCI}$, with no impact on the preoperative condition or postoperative outcomes. Hence, our home-based preoperative management for patients with HD may be effective and potentially feasible. This method may not be applicable to all cases; however, it can be considered as a preoperative management option for patients with HD. The danger of a fatal home HAEC is always there. Prompt recognition by parents is mandatory. The criteria for inclusion of patients for HCI should be clearly defined. Further studies with a larger number of $\mathrm{HCI}$ patients are required.

\section{Abbreviations \\ HD: Hirschsprung's disease; HAEC: Hirschsprung-associated enterocolitis; $\mathrm{HCl}$ : Home colorectal irrigation}

\section{Acknowledgements \\ We would like to thank Editage (www.editage.com) for providing writing support.}

\section{Authors' contributions}

T.S., M.N., and N.E. contributed to the conception and design of this study. T.S. collected data, performed the statistical analysis, and drafted the manuscript. N.E. critically reviewed the manuscript and supervised the whole study process. All authors read and approved the final manuscript.

\section{Funding}

There was no funding received for this study and the authors have no relevant financial relationships.

\section{Availability of data and materials}

The datasets used and/or analyzed during the current study are available from the corresponding author on reasonable request.

\section{Declarations}

Ethics approval and consent to participate

This study was approved by the ethics committee of Miyagi Children's Hospital (protocol identification number: 497). Written informed consent was obtained from the parents. 


\section{Consent for publication}

Not applicable.

\section{Competing interests}

The authors declare that they have no competing interests.

Received: 23 March 2021 Accepted: 9 July 2021

Published online: 12 August 2021

\section{References}

1. So HB, Schwartz DL, Becker JM, Daum F, Schneider KM. Endorectal "pullthrough" without preliminary colostomy in neonates with Hirschsprung's disease. J Pediatr Surg. 1980;15(4):470-1. https://doi.org/10.1016/S0022-34 68(80)80755-X

2. Cass DT. Neonatal one-stage repair of Hirschsprung's disease. Pediatr Surg Int. 1990;5:341-6.

3. Sulkowski JP, Cooper JN, Congeni A, Pearson EG, Nwomeh BC, Doolin EJ, et al. Single-stage versus multi-stage pull-through for Hirschsprung's disease: practice trends and outcomes in infants. J Pediatr Surg. 2014;49(11): 1619-25. https://doi.org/10.1016/j.jpedsurg.2014.06.002.

4. Hirose R, Kouhashi KI, Teshiba R, Yamada T, Hayashida Y. A new method of transanal catheter fixation for neonates with long segment-type Hirschsprung's disease. Surg Today. 2007;37(2):180-2. https://doi.org/10.1 007/s00595-006-3354-y.

5. Mochizuki K, Shinkai M, Kitagawa N, Take H, Usui H, Hosokawa T, et al. Continuous transanal decompression for infants with long- and total-type Hirschsprung's diseases as a bridge to curative surgery: a single-center experience. Surg Case Rep. 2017;3(1):42. https://doi.org/10.1186/s40792-0170318-y.

6. Lu C, Xie H, Li H, Geng Q, Chen H, Mo X, et al. Feasibility and efficacy of home rectal irrigation in neonates and early infancy with Hirschsprung disease. Pediatr Surg Int. 2019;35(11):1245-53. https://doi.org/10.1007/s003 83-019-04552-8.

7. Nakamura M, Wada M, Fukuzawa T, Tanaka H, Kudo H, Ando R, et al. Treatment of classic-type Hirschsprung's disease: rectoplasty with posterior triangular colonic flap versus transanal endorectal pull-through with rectoanal myotomy. Pediatr Surg Int. 2019;35(2):203-7. https://doi.org/10.1 007/s00383-018-4396-0.

8. Frykman PK, Kim S, Wester T, Nordenskjold A, Kawaguchi A, Hui TT, et al. Critical evaluation of the Hirschsprung-associated enterocolitis (HAEC) score: a multicenter study of 116 children with Hirschsprung disease. J Pediatr Surg. 2018:53(4):708-17. https://doi.org/10.1016/j.jpedsurg.2017.07.009.

9. Moore SW, Johnson G. Acetylcholinesterase in Hirschsprung's disease. Pediatr Surg Int. 2005;21(4):255-63. https://doi.org/10.1007/s00383-005-1383-z.

10. De Lorijn F, Kremer LCM, Reitsma JB, Benninga MA. Diagnostic tests in Hirschsprung disease: a systematic review. J Pediatr Gastroenterol Nutr. 2006;42(5):496-505. https://doi.org/10.1097/01.mpg.0000214164.90939.92.

11. Gosain A. Established and emerging concepts in Hirschsprung's-associated enterocolitis. Pediatr Surg Int. 2016;32(4):313-20. https://doi.org/10.1007/ s00383-016-3862-9.

12. Kyrklund K, Sloots CEJ, de Blaauw I, Bjornland K, Rolle U, Cavalieri D, et al. ERNICA guidelines for the management of rectosigmoid Hirschsprung's disease. Orphanet J Rare Dis. 2020;15(1):164. https://doi.org/10.1186/s13023020-01362-3.

13. Carcassonne M, Guys JM, Morisson-Lacombe G, Kreitmann B. Management of Hirschsprung's disease: curative surgery before 3 months of age. J Pediatr Surg. 1989;24(10):1032-4. https://doi.org/10.1016/S0022-3468(89)80209-X.

14. Lu C, Hou G, Liu C, Geng Q, Xu X, Zhang J, et al. Single-stage transanal endorectal pull-through procedure for correction of Hirschsprung disease in neonates and nonneonates: a multicenter study. J Pediatr Surg. 2017;52(7): 1102-7. https://doi.org/10.1016/j.jpedsurg.2017.01.061.

\section{Publisher's Note}

Springer Nature remains neutral with regard to jurisdictional claims in published maps and institutional affiliations. 